\title{
Disruption of the Corticoreticular Tract in Pediatric Patients With Trunk Instability: A Diffusion Tensor Tractography Study
}

\author{
Su Min Son, MD, So Min Shin, MD
}

Department of Physical Medicine and Rehabilitation, Yeungnam University College of Medicine, Daegu, Korea

\begin{abstract}
The authors report the diffusion tensor tractography (DTT) findings of three pediatric patients with gait dysfunction and corticoreticular tract (CRT) disruption. All three patients showed unilateral trunk instability, but they did not show any spasticity or weakness of the distal extremities. Clinical evaluation of trunk instability using a Trunk Control Measurement Scale (TCMS) revealed that the more affected side had a lower score than the contralateral side. DTT showed disrupted CRTs in hemispheres contralateral to the hemiparetic sides, which were associated with unilateral proximal instability, although conventional MRI showed no abnormal lesion explaining the hemiplegic symptom. Compared to the results in age-matched controls, these three patients had decreased values of fractional anisotropy (FA) and tract volumes (TV) of the affected CRTs, and these values were also decreased compared to those in the contralateral side. On the other hand, values of FA and TV of the corticospinal tracts on the ipsilateral and contralateral sides were only marginally different. In conclusion, diffusion tensor imaging can be helpful for investigating the state of the CRT in pediatric patients with trunk instability and gait dysfunction.
\end{abstract}

Keywords Diffusion tensor imaging, Corticoreticular tract, Gait dysfunction, Trunk instability, Pediatric patients

\section{INTRODUCTION}

Gait dysfunction is the most common problem in the pediatric rehabilitation field. Gait function is usually achieved between 12 and 14 months of age, and it consists of multiple components including neurological, mechanical, cognitive, and perceptual factors. However, the motor component is thought to be the most critical factor in gait function. The main motor pathways are classified as the corticospinal tract (CST, pyramidal tract) and nonCST tract (extrapyramidal tract). The CST is known to control voluntary movements of distal extremities, and it is known to be mainly related to fine motor skills [1]. On the other hand, gait function, which requires trunk

Received November 30, 2016; Accepted April 3, 2017

Corresponding author: So Min Shin

Department of Physical Medicine and Rehabilitation, Yeungnam University College of Medicine, 170 Hyeonchungno, Nam-gu, Daegu 42415, Korea. Tel: +82-53-620-3455, Fax: +82-53-620-3269, E-mail: nocturne27@naver.com

ORCID: Su Min Son (http://orcid.org/0000-0003-1185-1858); So Min Shin (http://orcid.org/0000-0003-1115-1704).

(c) This is an open-access article distributed under the terms of the Creative Commons Attribution Non-Commercial License (http://creativecommons.org/ licenses/by-nc/4.0) which permits unrestricted noncommercial use, distribution, and reproduction in any medium, provided the original work is properly cited. Copyright $\odot 2017$ by Korean Academy of Rehabilitation Medicine 
control and leg movement, is less associated with the CST and is more associated with the corticoreticular tract (CRT) [2]. The CRT originates from the premotor cortex (PMC) and terminates in the pontomedullary reticular formation. Several previous studies using conventional magnetic resonance imaging (MRI), single positron emission tomography-computed tomography (PET-CT), functional MRI (fMRI), and transcranial magnetic stimulation (TMS) have reported a strong association of PMC injury with gait dysfunction. Since the PMC is the main origin site of the CRT, it is considered that the CRT is one of the main neural pathways responsible for gait function [3].

Recent developments in diffusion tensor tractography (DTT) have enabled three-dimensional visualization and evaluation of neural tracts at the subcortical level. In 2012, Yeo et al. [4] identified the CRT in the human brain using DTT and a few other studies have reported CRT injury in patients with stroke or traumatic brain injury (TBI) [5-8]. However, little is known about the CRT status and its relation to gait dysfunction in pediatric patients who show delayed development.

In this study, we report three cases of pediatric patients with gait dysfunction, showing the corresponding DTT results.

\section{CASE REPORTS}

\section{Diffusion tensor imaging}

All three patients showed no definite abnormal findings on conventional brain MRI which does not explain the hemiplegic symptom of patients. Therefore, diffusion tensor imaging (DTI) was performed. DTI data were obtained using a synergy-L Sensitivity Encoding (SENSE) 6-channel head coil on a 1.5-T Philips Gyroscan Intera system (Hoffmann-La Roche, Best, The Netherlands).
We acquired 67 contiguous slices parallel to the anterior commissure-posterior commissure line. The imaging parameters used were as follows: matrix $=128 \times 128$, field of view $=221 \mathrm{~mm} \times 221 \mathrm{~mm}$, echo time $(\mathrm{TE})=76 \mathrm{~ms}$, repetition time (TR) $=10,726 \mathrm{~ms}$, SENSE factor (parallel imaging reduction factor) $=2$; EPI factor $=67$ and $b=1,000 \mathrm{~s} / \mathrm{mm}^{2}$, $\mathrm{NEX}=1$, and thickness $=2.3 \mathrm{~mm}$ (acquired voxel size 1.73 $\mathrm{mm} \times 1.73 \mathrm{~mm} \times 2.3 \mathrm{~mm}$ ).

The Oxford Center for Functional Magnetic Resonance Image of the Brain (FMRIB) Software Library (FSL; www. fmrib.ox.ac.uk/fsl) was used to analyze diffusion-weighted imaging data. Fiber tracking was performed using a probabilistic tractography method based on a multi-fiber model, and it was applied utilizing tractography routines implemented in FMRIB diffusion (5,000 streamline samples, $0.5 \mathrm{~mm}$ step lengths, curvature thresholds $=0.2$ ). The CST and CRT were identified by selecting fibers passing through both regions of interest (ROIs). A seed ROI of the CST was placed on the CST portion of the anterior mid-pons on a 2D color map. Target ROIs of the CST were located on the CST portion of the anterior low-pons on a 2D color map. To reconstruct the CRT, a seed ROI was positioned on the reticular formation in the medulla. The first target ROI was positioned on the midbrain tegmentum and the second target ROI was positioned on the PMC (Brodmann area 6) (Fig. 1). Values of fractional anisotropy (FA) and tract volume (TV) of the reconstructed CST and CRT were measured.

\section{Trunk Control Measurement Scale}

The Trunk Control Measurement Scale (TCMS) [9] was used to assess trunk stability. Laterality of trunk instability was evaluated by assessment of each trunk side. Total scores ranged from 0 (lowest performance) to 58 (best performance). Scores of the right and left sides ranged
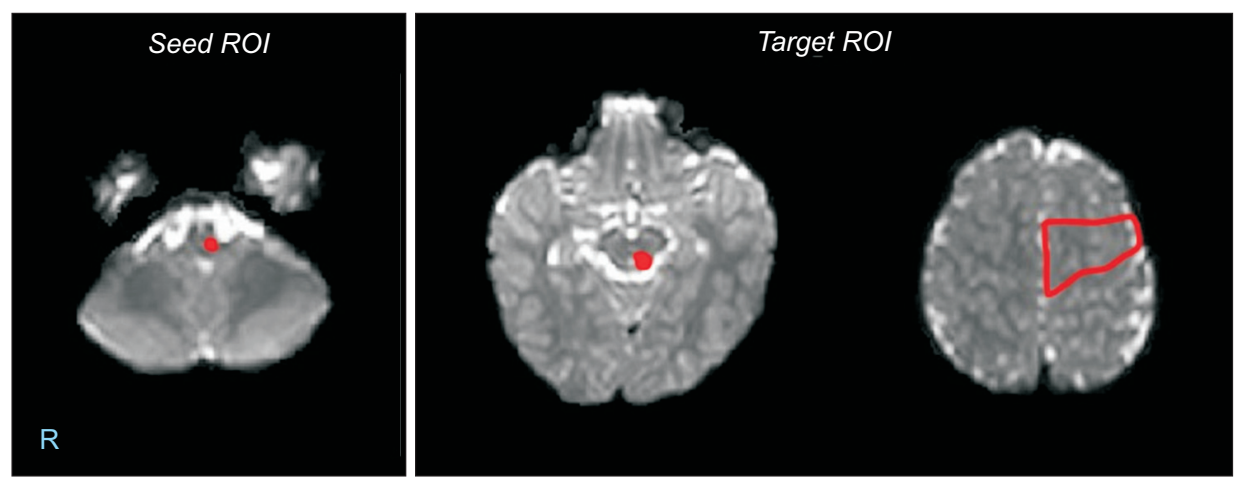

Fig. 1. Regions of interest (ROIs) were set for the corticoreticular tract (seed ROI, reticular formation in the medulla; first target ROI, midbrain tegmentum; second target ROI, Brodmann area 6). 
from 0 to 19, respectively, but the other scores showed no left or right bias. The reliability and validity of the TCMS in pediatric patients has been demonstrated in several previous studies [9].

Three pediatric patients who showed gait dysfunction and six age- and sex-matched typically developing controls were recruited into this study. All control subjects were volunteers whose parents had applied for participation in this study and the study provided research funds to control subjects. Written informed consent was obtained from the parents of all participants and the study was approved by the Institutional Review Board at Yeungnam University Medical Center (IRB No. PCR-1031). Demographic data and the functional data are summarized in Table 1. Statistical analyses were performed using the Statistical Package for Social Sciences (SPSS) ver. 23.0 (IBM Corp., Armonk, NY, USA). Demographic data was compared between the two groups using the Mann-Whitney test. The level of statistical significance was set at $\mathrm{p}<0.05$.

\section{Case 1}

This patient was born at a gestational age of $40+2$ weeks, and had a birth weight of $3.42 \mathrm{~kg}$, and no specific perinatal history. He could not stand independently when he visited our clinic at the age of 19 months. With someone holding his hand, he could barely take a few steps and he showed prominent trunk sway. His mother stated that he could stand better when she held his right hand rather than his left hand, that he could barely maintain the sitting position with his hands on the bottom at 12 months of age, and that he could not stand without assistance until 18 months of age. His crawling was not well controlled and he showed an intermediate level of creeping (crawling and belly) and crawling, but the hemiplegic pattern was not observed. He showed no definite spasticity, weakness or sensory problem in his distal extremities, but he exhibited right-side trunk instability. He used his left hand predominantly but showed poor fine motor coordination skills of both hands. His TCMS scores for the right and left sides were 1 and 6, respectively (Table 1). His conventional brain MRI showed no definite abnormal lesion that could explain the unilateral proximal instability and gait dysfunction. Values of FA and TV of bilateral CSTs showed only a marginal difference from those in controls (Table 2) and the integrity of bilat-

Table 1. Demographic and functional data of patients and control subjects

\begin{tabular}{lccccccccc}
\hline & P1 & P2 & P3 & C1a & C1b & C2a & C2b & C3a & C3b \\
\hline Age (mo) & 19 & 22 & 24 & 19 & 19 & 22 & 22 & 23 & 24 \\
Sex & M & M & M & M & M & M & M & M & M \\
Handedness & Rt & Rt & Rt & Rt & Rt & Rt & Rt & Rt & Rt \\
Birth weight (kg) & 3.42 & 3.14 & 3.6 & 3.48 & 3.42 & 3.29 & 3.54 & 3.57 & 3.37 \\
Gestational age (wk) & $40+2$ & $39+4$ & $41+0$ & $40+1$ & $39+5$ & $39+5$ & $40+2$ & $40+3$ & $39+2$ \\
Perinatal history & NS & NS & NS & NS & NS & NS & NS & NS & NS \\
Bayley & & & & & & & & & \\
MDI & 80 & 81 & 84 & - & - & - & - & - & - \\
PDI & 50 & 50 & 50 & - & - & - & - & - & - \\
TCMS & & & & & & & & & 58 \\
$\quad$ Total & 20 & 26 & 29 & 58 & 58 & 58 & 58 & 58 & $58 / 4$ \\
Static sitting (Rt/Lt/ND) & $1 / 4 / 3$ & $4 / 3 / 4$ & $4 / 4 / 4$ & $8 / 8 / 4$ & $8 / 8 / 4$ & $8 / 8 / 4$ & $8 / 8 / 4$ & $8 / 8 / 4$ & $8 / 8 / 4$ \\
Dynamic sitting (Rt/Lt/ND) & $0 / 2 / 10$ & $2 / 1 / 11$ & $2 / 1 / 12$ & $7 / 7 / 14$ & $7 / 7 / 14$ & $7 / 7 / 14$ & $7 / 7 / 14$ & $7 / 7 / 14$ & $7 / 7 / 14$ \\
Dynamic reaching (Rt/Lt/ND) & $0 / 0 / 0$ & $1 / 0 / 0$ & $1 / 0 / 1$ & $4 / 4 / 2$ & $4 / 4 / 2$ & $4 / 4 / 2$ & $4 / 4 / 2$ & $4 / 4 / 2$ & $4 / 4 / 2$ \\
\hline
\end{tabular}

P, patient; C, control; MDI, Mental Developmental Index; PDI, Psychomotor Development Index; GMFCS, Gross Motor Function Classification System; NS, non-specific; TCMS, Trunk Control Measurement Scale; Rt, right; Lt, left; ND, no directional bias.

There were no significant differences in the demographic data (age, birth weight, and gestational age) between the patient and control groups ( $p>0.05$ ). C1a and C1b were matched with P1, C2a and C2b were matched with P2, and C3a and C3b were matched with P3, respectively 
Table 2. CST parameters in patients and controls

\begin{tabular}{|ccccccccccc}
\hline CST & P1 & P2 & P3 & C1a & C1b & C2a & C2b & C3a & C3b \\
\hline $\begin{array}{c}\text { Fractional anisotropy } \\
\text { More affected-right }\end{array}$ & 0.432 & 0.463 & 0.458 & 0.460 & 0.438 & 0.515 & 0.528 & 0.541 & 0.531 \\
\hline $\begin{array}{l}\text { Less affected-left } \\
\text { Tract volume }\end{array}$ & 0.457 & 0.475 & 0.462 & 0.481 & 0.444 & 0.482 & 0.516 & 0.461 & 0.509 \\
\hline $\begin{array}{l}\text { More affected-right } \\
\text { Less affected-left }\end{array}$ & 518 & 557 & 610 & 453 & 529 & 617 & 589 & 784 & 790 \\
\hline
\end{tabular}

C1a and C1b were matched with P1; C2a and C2b were matched with P2; and C3a and C3b were matched with P3, respectively.

P, patient; C, control; CST, corticospinal tract; More affected-right, right side for controls and the more affected side for patients; Less affected-left, left side for the controls and the less affected side for patients.

A

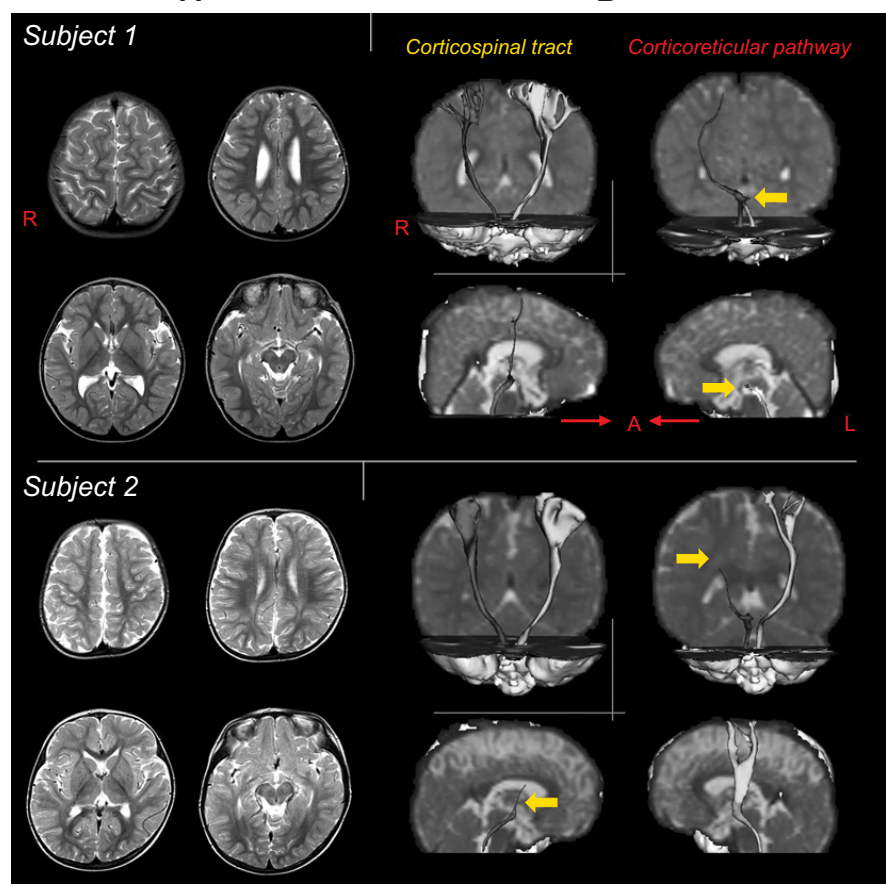

A

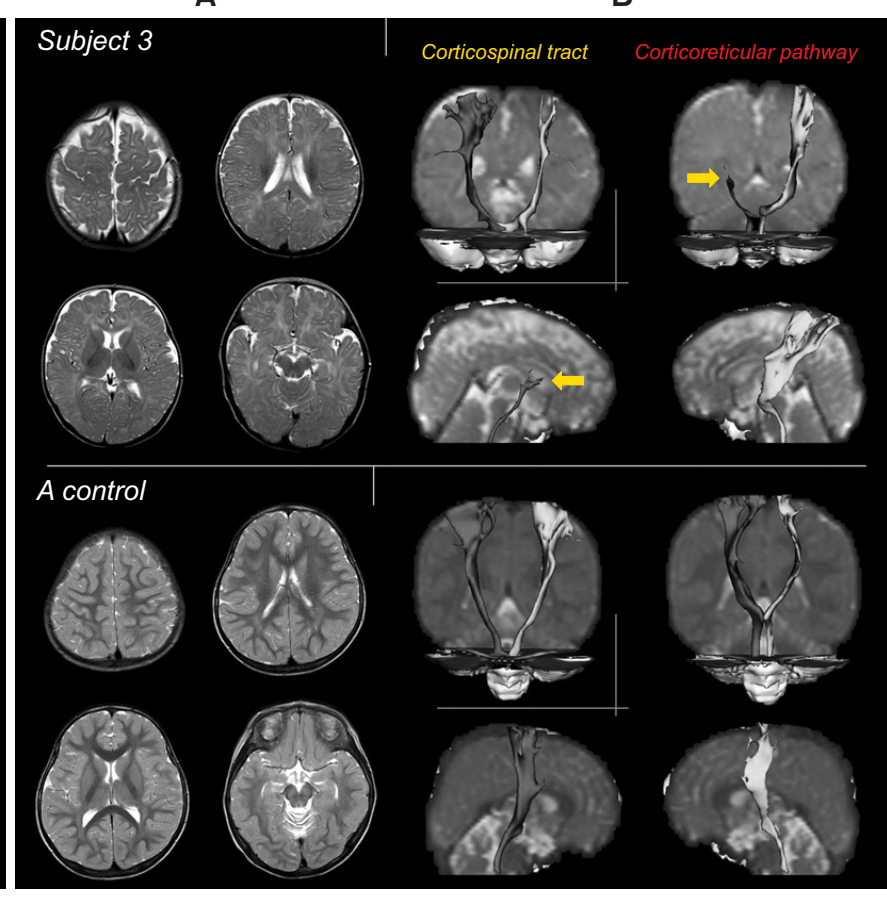

Fig. 2. (A) T2-weighted axial magnetic resonance images. (B) Diffusion tensor tractography of the corticospinal tract (CST) and the corticoreticular tract (CRT). Bilateral CSTs were preserved in all three patients. Subject 1 showed disruption of the left CRT, whereas subjects 2 and 3 showed disruption of the right CRTs. Control subjects showed preserved integrity of the CST and CRT.

eral CSTs was preserved (Fig. 2). However, the left CRT showed disrupted integrity (Fig. 2). DTT result of bilateral CRTs showed decreased values of FA and TV compared to those in controls (Table 3). Other neural tracts involved in motor function such as the medial lemniscus and cerebellar peduncles were also analyzed and no abnormal findings were found.

\section{Case 2}

This patient was born at a gestational age of 39+4 weeks and had a birth weight of $3.14 \mathrm{~kg}$. At the age of 22 months, the patient and his mother visited our rehabilitation department due to concerns about his delayed development. He could not walk independently despite his age. Interestingly, he could walk when his mother held his left hand, although he exhibited trunk sway. However, he was unable to walk when his mother held his right hand 
Table 3. CRT parameters in patients and controls

\begin{tabular}{|cccccccccc}
\hline \multicolumn{1}{c}{ CRT } & P1 & P2 & P3 & C1a & C1b & C2a & C2b & C3a & C3b \\
\hline Fractional anisotropy & & & & & & & & & \\
$\quad$ More affected-right & 0.286 & 0.356 & 0.404 & 0.516 & 0.521 & 0.550 & 0.535 & 0.570 & 0.562 \\
\hline Less affected-left & 0.397 & 0.458 & 0.434 & 0.512 & 0.512 & 0.540 & 0.539 & 0.539 & 0.538 \\
\hline Tract volume & & & & & & & & & \\
$\quad$ More affected-right & 75 & 264 & 329 & 468 & 451 & 687 & 682 & 820 & 878 \\
\hline Less affected-left & 284 & 448 & 561 & 421 & 468 & 713 & 732 & 892 & 889 \\
\hline
\end{tabular}

$\mathrm{Cla}$ and $\mathrm{Clb}$ were matched with P1; C2a and C2b were matched with P2; and C3a and C3b were matched with P3, respectively.

P, patient; C, control; CRT, corticoreticular tract; More affected-right, right side for controls and the more affected side for patients; Less affected-left, left side for the controls and the less affected side for patients.

due to severe trunk instability. His mother described his delayed developmental milestones as follows: he could maintain the neck posture at the age of 5 to 6 months, and he could not stand alone until the age of 15 months. He did not have a specific hemiplegic pattern during crawling. His right and left side TCMS scores were 7 and 4, respectively (Table 1). DTT showed right CRT disruption (Fig. 2) and decreased values of FA and TV of bilateral CRTs compared to those in controls (Table 3). Bilateral CSTs showed preserved integrity (Fig. 2) and the values of FA and TV showed no definite difference from those in controls (Table 2). The medial lemniscus and cerebellar peduncles showed no abnormal findings.

\section{Case 3}

This male patient was referred to our rehabilitation department for delayed development at the age of 24 months. He was born at $41+0$ weeks and had a birth weight of $3.6 \mathrm{~kg}$. His parents described clinical manifestations which were similar to those of the second patient, i.e., he could only walk with left side assistance and showed no specific hemiplegic pattern during crawling. He showed right hand dominance, but, he had impaired fine motor coordination skills of both hands. In addition, he exhibited trunk instability even when standing while holding his mother's hand. Like the first patient, the second and third patients showed no spasticity or weakness of their distal extremities. His right and left TCMS scores were 7 and 5, respectively (Table 1). Like the first and second patients, the right CRT of the third patient showed disruption and the left CRT and bilateral CSTs showed no evidence of disruption (Fig. 2). DTT of bilateral CRTs, especially the right side, showed decreased values of FA and TV compared to those in controls (Table 3), but, the values of bilateral CSTs showed only a marginal difference from those in controls (Table 2). Similarly, the medial lemniscus and cerebellar tracts showed no definite abnormal findings explaining the patient's symptom.

\section{DISCUSSION}

Here, we report three cases of pediatric patients with gait dysfunction and trunk instability that appeared attributable to CRT disruption.

Several studies have reported CRT injury in patients with stroke or traumatic brain injury. In 2013, Yeo and Jang [5] reported that improvement of proximal and axial motor weakness was related to the recovery of an injured CRT in a patient with intracerebral hemorrhage. Furthermore, they concluded that proximal weakness in patients with traumatic brain injury is mainly due to CRT injury, rather than CST injury [6]. There was another DTT study in patients with cerebral infarct. Do et al. [7] recruited four hemiparetic patients with cerebral infarct who showed severe proximal weakness rather than distal weakness. In their DTT results, the affected CST showed preserved integrity in all patients. However, on the contrary, the affected CRT showed Wallerian degeneration in two patients and prominent disruption at the infarct level in the other two patients. In 2015, Lee and Jang [8] recruited 29 patients with proximal weakness following mild TBI and 25 control subjects. They showed that the TV of the CRT was significantly decreased in the patient group than in the control group. Similarly, in our patients, despite unilateral proximal weakness, there was no significant difference in CST parameters between right 
and left sides, or between patients and control subjects. However, the more affected CRT showed definite disruption and decreased DTI parameters compared to the less affected CRT, although conventional MRI showed no definite abnormal lesion. These results suggest that the CRT plays an important role in gait function and trunk stability, and that DTI could be helpful for assessment of the CRT in pediatric patients with gait dysfunction and trunk instability.

There are no published papers on the CRT problem in pediatric patients, and the cause of CRT disruption in pediatric patients has not yet been elucidated. There is one published paper on the normal maturation pattern of the CST and CRT using DTT. In 2014, Yeo et al. [10] investigated maturation of the CST and CRT in 75 normal healthy subjects ranging in age from 3 months to 25 years using DTI. In their study, all participants including infants, showed preserved integrity of the CST and CRT despite their young age. This indicates that disruption of the CRT in our patients cannot be considered a normal developmental process in the immature brain. Moreover, all patients included in our report showed unilateral disruption of the CRT. During normal development, neural tracts associated with motor function mature with symmetric integrity. The CRT is also a neural tract associated with motor function. Asymmetric integrity and unilateral disruption of the CRT in our patients seems difficult to be understood as a normal developmental process. Considering the absence of lesions on conventional brain MRI, the authors think that unilateral CRT disruption in our patients may be due to microstructural damage or pathological immaturity. Therefore, our results about the CRT are thought to have a meaningful relation with the pathological state of unilateral trunk instability.

However, several limitations of this study should be considered. Firstly, because the present study is essentially a case report, generalizability of its findings is limited. Another limitation is with respect to the TCMS measurement. In previous studies, TCMS was used in pediatric patients older than 5 years. The authors were aware of this fact; however, to the best of our knowledge, TCMS is the only measurement scale that enables assessment of unilateral trunk stability, and therefore, we used the TCMS. This is another limitation of this study. Evidently, large-scale studies with more detailed clinical evaluations are warranted.

\section{CONFLICT OF INTEREST}

No potential conflict of interest relevant to this article was reported.

\section{ACKNOWLEDGMENTS}

This work was supported by a grant from Chunma medical research foundation, Korea, 2016.

\section{REFERENCES}

1. Lessek AM. The pyramidal tract; basic considerations of corticospinal neurons. Res Publ Assoc Res Nerv Ment Dis 1948;27:106-28.

2. Matsuyama K, Mori F, Nakajima K, Drew T, Aoki M, Mori S. Locomotor role of the corticoreticular-reticulospinal-spinal interneuronal system. Prog Brain Res 2004;143:239-49.

3. Freund HJ, Hummelsheim H. Premotor cortex in man: evidence for innervation of proximal limb muscles. Exp Brain Res 1984;53:479-82.

4. Yeo SS, Chang MC, Kwon YH, Jung YJ, Jang SH. Corticoreticular pathway in the human brain: diffusion tensor tractography study. Neurosci Lett 2012;508:912.

5. Yeo SS, Jang SH. Recovery of an injured corticospinal tract and an injured corticoreticular pathway in a patient with intracerebral hemorrhage. NeuroRehabilitation 2013;32:305-9.

6. Yeo SS, Kim SH, Jang SH. Proximal weakness due to injury of the corticoreticular pathway in a patient with traumatic brain injury. NeuroRehabilitation 2013;32: 665-9. 23648621.

7. Do KH, Yeo SS, Lee J, Jang SH. Injury of the corticoreticular pathway in patients with proximal weakness following cerebral infarct: diffusion tensor tractography study. Neurosci Lett 2013;546:21-5.

8. Lee HD, Jang SH. Injury of the corticoreticular pathway in patients with mild traumatic brain injury: a diffusion tensor tractography study. Brain Inj 2015;29: 1219-22.

9. Heyrman L, Molenaers G, Desloovere K, Verheyden G, De Cat J, Monbaliu E, et al. A clinical tool to measure trunk control in children with cerebral palsy: the Trunk Control Measurement Scale. Res Dev Disabil 
2011;32:2624-35.

10. Yeo SS, Jang SH, Son SM. The different maturation of the corticospinal tract and corticoreticular pathway in normal brain development: diffusion tensor imaging study. Front Hum Neurosci 2014;8:573. 